\title{
Community Attachments are Associated with COVID-19 Public Health Behaviors Among Adolescents in Pakistan
}

\author{
Faiza Nisar ${ }^{1} \cdot$ Sadaf Zeb ${ }^{1} \cdot$ Benjamin Oosterhoff ${ }^{2} \cdot$ Shaf Ahmed $^{3}$
}

Accepted: 28 September 2021 / Published online: 11 October 2021

(C) The Author(s), under exclusive licence to Springer Science+Business Media, LLC, part of Springer Nature 2021

\begin{abstract}
Background Community attachments are thought to promote adolescents' engagement in public health behaviors. To date, past research has exclusively examined the social benefits of community attachments among adolescents in the United States and less is known about these benefits among youth in low-income adolescent-dense countries such as Pakistan.

Objective The present study examined associations between Pakistani adolescents' community attachments and COVID-19 public health behaviors, including social distancing, disinfecting, hoarding, news monitoring.

Method Adolescents living in Pakistan $(\mathrm{N}=1,110 ; 13-18$ years; $\mathrm{M}=16.70)$ reported on their COVID-19 public health behavior (social distancing, disinfecting behaviors, hoarding behaviors, news monitoring) and community attachments (social responsibility values, social trust, self-interest values).

Results Greater social responsibility values were associated with greater social distancing $(B=.09, p=.009)$ and disinfecting behavior $(B=.39, p<.001)$. Greater social trust was significantly associated with greater disinfecting $(B=.09, p<.001)$ and greater hoarding behaviors $(B=.07, p=.001)$ and greater self-interest values were associated with lower social distancing $(B=-.06, p=.010)$, greater disinfecting $(B=.15, p<.001)$, and greater hoarding behaviors $(B=.11, p=.001)$.

Conclusion Results from this study demonstrate that community attachments may play an important role in guiding adolescents' public health behavior in Pakistan. These findings extend past research and contribute to an inclusive and culturally sensitive model of the benefits of adolescents' community attachments for public heatlh.
\end{abstract}

Keywords COVID-19 $\cdot$ Adolescence $\cdot$ Community attachments $\cdot$ Social responsibility

Benjamin Oosterhoff

Benjamin.oosterhoff@montana.edu

1 Department of Professional Psychology, Bahria University Islamabad, Islamabad, Pakistan

2 Department of Psychology, Montana State University, P.O. Box 173440, Bozeman, MT 59717-3440, USA

3 Department of Behavioral Sciences, National University of Science and Technology, Islamabad, Pakistan 
Psychologists and community health professionals have had longstanding interest in promoting adolescent public health behavior (Wray-Lake et al., 2012). Adolescence represents the developmental period between 13 and 18 years and is characterized by the co-occurance of physical, psychological, and social changes that produce a sensitive period for the adoption of several health-related behaviors (Sawyer et al., 2012). Theoretical models of adolescent public health have emphasized the importance of community attachmentsdefined as high social responsibility, high social trust, and low self-interest—as individual differences that predict personal and social health behaviors (Oosterhoff \& Palmer, 2020; Wray-Lake et al., 2012). Youth who feel more attached to their community and demonstrate higher social responsibility, higher social trust, and lower self-interest may be more concerned with protecting themselves and others, and thus engage greater public health behaviors (Wray-Laket et al., 2012). Past research has shown that adolescents with high community attachments were less engaged in substance use (Wray-Lake et al., 2012) and were more engaged in prosocial behavior during the COVID-19 pandemic (Oosterhoff \& Palmer, 2020) relative to those with low community attachments. To date, research on the public health implications of adolescents' community attachments has almost exclusively focused on youth from the United States. Less is known about whether community attachments are similarly connected to positive public health behaviors within different cultural contexts, including lower income countries with a high proportion of young people such as Pakistan. Examining connections between adolescents' community attachments and public health behaviors among youth from non-Western cultures is critical to testing the generalizability of these models and thus represents a logical next step in advancing theory on adolescent public health.

The COVID-19 pandemic provides a unique opportunity to understand whether and how community attachements may be linked with public health behavior among Pakasatni youth. Like many countries, Pakistan enacted several social policies to combat the spread of COVID-19. Citizens were encouraged to practice social distancing by remaining at least 6 feet apart from one another. Furthermore, all public areas including shopping malls, parks, restaurants, and educational institutions were closed and a strict lockdown was implemented. Citizens were encouraged to practice good hygiene by washing hands frequently and using disinfectant. The widespread fear that accompanied COVID19 also lead some people to become concerned about the availability of basic necessities and to engage in panic buying or hoarding of food products and other supplies. Further, the rapidly changing nature of the disease requires that citizens remain current on the latest news about the pandemic. Together, these challenges highlight the importance of social distancing, disinfecting behaviors, COVID-19 news monitoring, and descreased hoarding behaviors for public health. Understanding variability in these behaviors may be especially important among Pakastani youth, as Pakistan is a predominantly young country with almost $50 \%$ of the population are children and adolescents (Imran et al., 2020).

To date, only one study has examined links between community attachments and adolescents' responses to COVID-19 among youth in the United States (Oosterhoff \& Palmer, 2020). Adolescents who had higher social responsibility, higher social trust, and lower self-interest generally engaged in greater social distancing, greater disinfecting behavior, greater news monitoring, and lower hoarding behavior, although these links were nuanced. Examining these connections among youth from non-Western cultures is necessary to advance theory on public health (van Bavel et al., 2020) and may be especially important for research examining the benefits of community attachments, which may greatly differ across types of communities and have limited generalizability across cultural contexts. 
The goal of this study was to examine connections between community attachments and adolescents' responses to COVID-19 among Pakistani youth. Based on past research (Oosterhoff \& Palmer, 2020), we hypothesized that greater social responsibility and social trust would be associated with greater news monitoring, greater social distancing, and greater disinfecting behaviors whereas greater self-interest values would be associated with more hoarding behaviors among Pakistani adolescents during COVID-19 pandemic. Given that Oosterhoff et al. (2020) also examined beliefs about severity of COVID-19, we tested whether these beliefs were similarity associated with youths' COVID-19 response in a Pakistani sample.

\section{Method}

\section{Sample}

This study included 1,110 adolescents living in Pakistan (13-18 years; $M=16.70$, $S D=1.39)$. The sample was comprised of youth in 9 th grade $(9.8 \%), 10$ th grade $(24.8 \%)$, 11 th grade $(24.3 \%)$, 12th grade $(3.7 \%)$, college $(37.1 \%)$, and not in school $(0.3 \%)$. The sample included more female participants $(72.9 \%)$ as compared to male participants (27.1\%). Youth primarily identified as Punjabi (62\%), followed by Pakhtun (13.8\%), Sindhi (1.0\%), Baloch (0.4\%), Hindkowans (6.3\%), Kashmiri (7.4\%) and others (9.2\%). Participants reported their family's financial strain (Metzger et al., 2018) by indicating whether their families were able to buy all the things they wish/desire $(17.3 \%)$, having no problem buying things that are needed or even buy some more extra things (47.1\%), having just enough money to be able to buys things that are needed $(29.1 \%)$, and having a difficult time purchasing things they need $(6.5 \%)$.

\section{Procedure}

Data was collected from 5 to $15^{\text {th }}$ April of 2020 from 8:00 AM until 5:00 PM. The online survey was advertised on different social media platforms (Instagram, Facebook, WhatsApp and though email). Only participants who had internet and mobile phones were invited to participate in this study. After providing informed consent, adolescents selected into the study and completed a 5-min online survey. This study was approved by the Institutional Review Board of the first author's institution and the authors declare no conflicts of interest.

\section{Instruments}

\section{Demographic Characteristics}

Participants reported their age, gender, ethnicity/race, parental education, and family financial strain prior to the other measures. Participants parental education was recoded on a 3-point scale from 1 (neither parent completed high school), 2 (at least one parent complete high school) and 3 (at least one parent completed college). Participants recorded their political ideology on 5-point Likert scale from 1 (very conservative) to 5 (very liberal) along with another choice to depict 'I don't know'. 


\section{Social Distancing}

Five items $(\alpha=0.67)$ developed by Oosterhoff et al. (2020) were used to measure social distancing. Participants rated the frequency at which they spent time in person with the extended family, neighbors, teachers, friends and other people who do not reside with them during the past week (7 days) on a 5-point Likert scale from 1 (not at all) to 5 (very often). Mean scores were calculated with values indicating greater social distancing.

\section{Disinfecting Behaviors}

Four items $(\alpha=0.64)$ developed by Oosterhoff et al. (2020) were used to measure disinfecting behaviors. Participants rated the amount of time in which they cleaned their cellphones, washed their hands, and used disinfecting wipes during the past week (7 days) on a 6-point Likert scale from 1 (not at all) to 6 (multiple times a day). Mean scores were calculated with higher values indicating more disinfecting behavior.

\section{Hoarding Behaviors}

A single item created by Oosterhoff et al. (2020) was used to assess hoarding behavior. Participants rated how often they had hoarded supplies from a department or grocery shops in the past week on a 5-point Likert scale from 1 (not at all) to 5 (very often). Higher values indicated greater hoarding behaviors.

\section{COVID-19 News Monitoring}

A single item was used to assess COVID-19 news monitoring. Participants rated the extent to which they have followed the latest news coverage about COVID-19. Participants responded on a 5-point Likert scale from 1 (not at all) to 5 (a great deal). Higher values indicated greater COVID-19 news monitoring.

\section{Attitudes about COVID -19 Severity}

Four items $(\alpha=0.67)$ created by Oosterhoff et al. (2020) were used to measure attitudes about COVID-19 severity (e.g., "The Coronavirus is just 'Flu'”). Responses were given on a 7-point Likert scale from 1 (strongly disagree) to 7 (strongly agree). Mean scores were calculated, with higher values indicating greater beliefs about the severity of COVID-19.

\section{Social Responsibility Values}

Three items $(\alpha=0.74)$ taken from Oosterhoff et al. (2020) were used to measure social responsibility values. Participants rated the importance of considering the needs of other persons, assure that other persons are being treated justly, and imagine how other people are affected by their actions in future. Responses were given on a 5-point Likert scale 
from 1 (not at all important) to 5 (extremely important). Mean scores were calculated with higher values indicating the high level of social responsibility values.

\section{Social Trust}

Three items $(\alpha=0.80)$ used by Oosterhoff et al. (2020) were used to measure social trust. Participants rated their agreement with a series of statements (e.g., 'Most people can be trusted') to measure the social trust on a 7-point Likert scale from 1 (strongly disagree) to 7 (strongly agree). Mean scores were calculated with higher values indicating greater social trust.

\section{Self-Interest Values}

Self-interest values were measured using 2-items $(r=0.50)$ used by Oosterhoff et al. (2020). Participants rated the importance of putting their own needs before the needs of other people, and do what they would like, no matter what other people want. Participants responded on a 5-point Likert scale from 1 (not at all important) to 5 (extremely important). Mean scores were calculated with higher values indicating greater self-interest values.

\section{Results}

Frequencies of news monitoring, social distancing, disinfecting, and hoarding behaviors over the past 7 days are displayed in the supplemental file. During this period, only $0.9 \%$ of youth engaged in complete social distancing, with no in-person contact with anyone outside of their household. About $4.1 \%$ youth reported engaging in one type of disinfecting behaviors in multiple times a day. Moreover, a small proportion of youth (24.1\%) engaged in a little hoarding within the past week. About $21.9 \%$ youth indicated that they did not follow news related to COVID-19, whereas (75.9\%) following the COVID-19 news at least a little.

Table 1 displays correlations, means, and standard deviations for all study variables. Social responsibility values were positively correlated with attitudes about the severity of COVID-19, social trust, self-interest values, social distancing, and disinfecting behavior. Attitudes about severity of COVID-19 were positively correlated with social distancing and negatively correlated with social trust, hoarding behavior, and COVID-19 news monitoring. Social trust was positively correlated with self- interest, disinfecting behavior, and hoarding behaviors and negatively correlated with social distancing. Self-interest values were positively correlated with with disinfecting and hoarding behaviors. Social distancing was negatively correlated with disinfecting and hoarding behaviors. Disinfecting behaviors was positively correlated with hoarding behaviors.

Tables 2 and 3 display regression models predicting adolescent social distancing, disinfecting, hoarding, and news monitoring behaviors and Fig. 1 shows scatterplots of the significant associations. Adolescent girls, older youth, those with stronger attitudes about the severity of COVID-19, those with higher social responsibility values, and those with lower self-interest values engaged in greater social distancing. Overall, this model accounts for $10 \%$ of the variance in social distancing. Additionally, lower financial strain, greater parents' education, greater social responsibility values, greater social trust, and greater self-interest were significantly associated with greater disinfecting behavior. This model 





Table 2 Regression models predicting adolescent social distancing and disinfecting behaviors

\begin{tabular}{|c|c|c|c|c|c|c|c|c|}
\hline & \multicolumn{4}{|c|}{ Social distancing } & \multicolumn{4}{|c|}{ Disinfecting behavior } \\
\hline & $\mathrm{B}$ & SE & $95 \% \mathrm{CI}$ & $\mathrm{p}$ & $\mathrm{B}$ & SE & $95 \% \mathrm{CI}$ & $\mathrm{p}$ \\
\hline \multicolumn{9}{|l|}{ Demographics } \\
\hline Gender & 0.22 & 0.04 & $0.13-0.30$ & $<0.001$ & 0.04 & 0.07 & $-0.09-0.17$ & 0.558 \\
\hline Age & 0.03 & 0.01 & $0.00-0.06$ & 0.034 & -0.03 & 0.02 & $-0.07-0.01$ & 0.196 \\
\hline Ethnicity & 0.03 & 0.04 & $-0.05-0.11$ & 0.451 & 0.09 & 0.06 & $-0.03-0.20$ & 0.152 \\
\hline Financial status & 0.01 & 0.02 & $-0.03-0.06$ & 0.557 & -0.09 & 0.04 & -0.16 to -0.02 & 0.012 \\
\hline Parents' education & 0.04 & 0.03 & $-0.01-0.10$ & 0.128 & 0.14 & 0.04 & $0.06-0.23$ & 0.001 \\
\hline Ideology & 0.04 & 0.02 & $-0.01-0.08$ & 0.141 & 0.02 & 0.04 & $-0.05-0.09$ & 0.616 \\
\hline \multicolumn{9}{|l|}{ COVID attitudes } \\
\hline Covid attitudes & 0.12 & 0.02 & $0.08-0.15$ & $<0.001$ & 0.01 & 0.02 & $-0.03-0.06$ & 0.569 \\
\hline \multicolumn{9}{|c|}{ Community attachments } \\
\hline $\begin{array}{l}\text { Social responisibil- } \\
\text { ity values }\end{array}$ & 0.09 & 0.04 & $0.02-0.16$ & 0.009 & 0.39 & 0.05 & $0.29-0.50$ & $<0.001$ \\
\hline Social trust & -0.00 & 0.01 & $-0.03-0.02$ & 0.753 & 0.09 & 0.02 & $0.05-0.14$ & $<0.001$ \\
\hline Self interest values & -0.06 & 0.02 & -0.10 to -0.01 & 0.010 & 0.15 & 0.03 & $0.08-0.22$ & $<0.001$ \\
\hline $\mathrm{R}^{2} / \mathrm{R}^{2}$ adjusted & \multicolumn{4}{|c|}{$0.096 / 0.088$} & \multicolumn{4}{|c|}{$0.110 / 0.102$} \\
\hline
\end{tabular}

$* p<.05, * * p<.01$. Bolded values area significant at $p<.05$

Table 3 Regression models predicting adolescent hoarding behaviors and news monitoring

\begin{tabular}{|c|c|c|c|c|c|c|c|c|}
\hline & \multicolumn{4}{|c|}{ Hoarding Behavior } & \multicolumn{4}{|c|}{ News Monitoring } \\
\hline & $\mathrm{B}$ & SE & $95 \% \mathrm{CI}$ & $\mathrm{p}$ & B & SE & $95 \% \mathrm{CI}$ & $\mathrm{P}$ \\
\hline \multicolumn{9}{|l|}{ Demographics } \\
\hline Gender & -0.18 & 0.06 & -0.31 to -0.05 & 0.005 & -0.17 & 0.08 & -0.33 to -0.01 & $\mathbf{0 . 0 3 7}$ \\
\hline Age & -0.01 & 0.02 & $-0.05-0.03$ & 0.555 & 0.03 & 0.03 & $-0.02-0.08$ & 0.270 \\
\hline Ethnicity & -0.01 & 0.06 & $-0.13-0.10$ & 0.819 & -0.05 & 0.07 & $-0.20-0.10$ & 0.496 \\
\hline Financial Status & -0.10 & 0.04 & -0.17 to -0.03 & 0.004 & 0.00 & 0.05 & $-0.09-0.09$ & 0.931 \\
\hline Parents' Education & 0.00 & 0.04 & $-0.08-0.08$ & 0.947 & 0.09 & 0.05 & $-0.01-0.19$ & 0.084 \\
\hline Ideology & -0.08 & 0.04 & -0.15 to -0.00 & 0.036 & -0.00 & 0.05 & $-0.09-0.09$ & 0.964 \\
\hline \multicolumn{9}{|l|}{ COVID attitudes } \\
\hline COVID attitudes & -0.04 & 0.02 & $-0.09-0.00$ & 0.068 & 0.07 & 0.03 & $0.01-0.13$ & 0.016 \\
\hline \multicolumn{9}{|c|}{ Community attachments } \\
\hline Social Resp. & 0.06 & 0.05 & $-0.04-0.16$ & 0.246 & 0.07 & 0.07 & $-0.06-0.20$ & 0.263 \\
\hline Social Trust & 0.07 & 0.02 & $0.03-0.12$ & 0.001 & 0.00 & 0.03 & $-0.05-0.06$ & 0.878 \\
\hline Self Interest & 0.11 & 0.03 & $0.05-0.18$ & 0.001 & -0.08 & 0.04 & $-0.16-0.01$ & 0.079 \\
\hline $\mathrm{R}^{2} / \mathrm{R}^{2}$ adjusted & \multicolumn{4}{|c|}{$0.054 / 0.046$} & \multicolumn{4}{|c|}{$0.017 / 0.008$} \\
\hline
\end{tabular}

$* p<.05, * * p<.01$. Bolded values area significant at $p<.05$

accounted for $11 \%$ of the variance in disinfecting behavior. With regards to hoarding behavior, adolescent boys, those with greater financial strain, those who were more conservative, and those with higher social trust and self-interest engaged in greater hoarding behavior. This model accounted for $6 \%$ of the variance in hoarding behavior. For news 

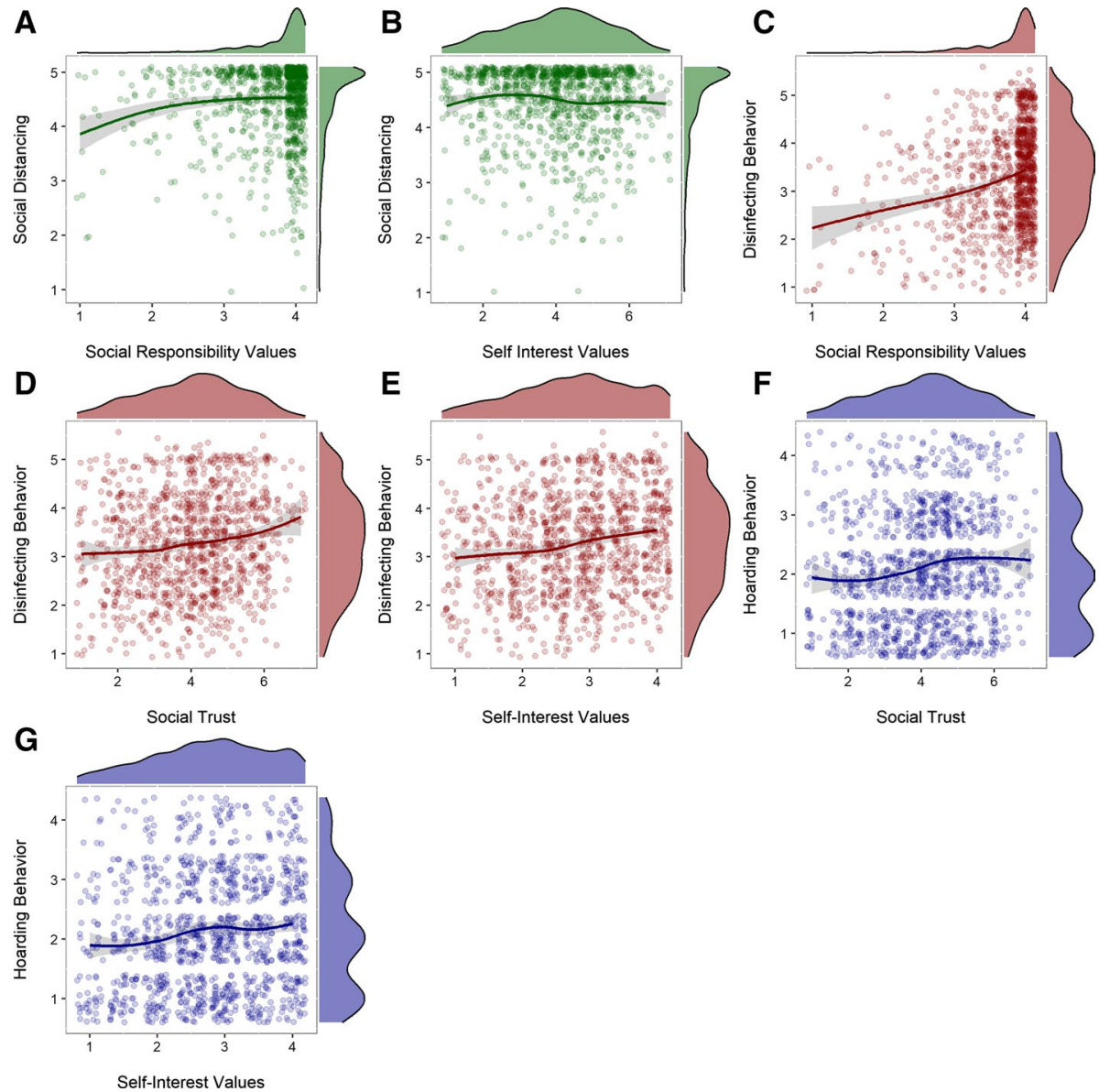

Self-Interest Values

Social Trust

Fig. 1 a Association between social responsibility values and social distancning. b Association between self-interest values and social distancing. c Association between social responsibility values and disinfecting behavior. d Association between social trust and disinfecting behavior. e Association between self-interest values and disinfecting behavior. f Association between social trsut and horading behavior. g Association between self-interest values and hoarding behavior

monitoring, adolescent boys and those who viewed COVID-19 as more serious engaged in greater news monitoring. This model accounted for $2 \%$ of the variance in news monitoring.

\section{Discussion}

Community attachments are theorized to represent an important individual difference in adolescents' public health behavior, yet little research has examinined the benefits of community attachments among non-US youth (Wray-Lake et al., 2012). The present study examined associations among adolescents' community attachments and news monitoring, social distancing, disinfecting and hoarding behaviors among Pakistani youth during the COVID-19 pandemic. Results indicate that adolescents with stronger community attachments generally engaged in greater COVID-19 health-related behaviors. These findings 
were consistent after accounting for a range of demographic characteristics and attitudes about COVID-19 severity.

Several findings from this study were consistent with past research with U.S. adolescents (Oosterhoff \& Palmer, 2020). Similar to Oosterhoff and Palmer (2020), our results indicate that greater social responsibility was associated with more disinfecting behavior, and greater self-interest values were associated with lower social distancing, more hoarding behavior, and more disinfecting behavior. Overall, this pattern of results indicate that four out of seven statistically significant associations between community attachments and adolescents' behavioral response to COVID-19 were present among Pakistani adolescents approximately one month after COVID-19 was declared a global pandemic. These findings provide important information regarding the generalizability of community attachments for public health behavior. To date, the vast majority of research on the benefits of community attachments has been conducted with US samples (e.g., Wray-Lake et al., 2012). The nearly complete focus of community attachments within the US context may produce a collider bias by design due to unique cultural characteristics of adolescents and their communities within the US. Findings from this study extend past research on the importance of community attachments for community and adolescent health by demonstrating that these links partially generalize among youth in Pakistan - a low income country with a high proportion of young people. Such extensions are especially important within the context of global pandemics when the threat to global health requires collective action.

There were some notable discrepancies from this study compared to past research (Oosterhoff \& Palmer, 2020). Unlike past research, we found that greater social responsibility values were associated with greater social distancing and we did not find significant associations between social responsibility values and news monitoring or hoarding. Additionally, past research found significant associations between greater social trust and less hoarding behavior, whereas the opposite pattern was found in this study. Further, greater self-interest was positively correlated with social responsibility values in this study, whereas the opposite pattern has been found in past research (Oosterhoff \& Palmer, 2020). Discrepancies between past research and the current study may be due to multiple factors. One important confound between the two studies concerns the progression of the virus and when data were collected. Oosterhoff and Palmer (2020) collected data from youth in late March 2020, shortly after COVID-19 was declared a national emergency in US. In contrast, data in this study was collected over the month of April 2020. It is possible that selfinterest remained a pertinent motivator for COVID-19 related behavior as the virus spread. In contrast, social responsibility may have been higher and a more central motivator early on in the pandemic, and became less relevant in the month following. This possibility is consistent with past research that has shown that community attachments may increase in the immediate aftermath of disasters (Kang \& Skidmore, 2018).

Discrepancies between findings from this study and past research may also be due to cultural differences related to the role of community attachments and pandemic-related responses. The prevailing poverty in Pakistan has created several budgetary and logistical challenges for the government to meet the needs of those with fewer resources. Those with the resources to hoard supplies may only do so if they feel enough social trust as to not incur personal or social retaliation for hoarding when many are faced with high poverty rates. Additionally, religiousity is an important motivator for participating in prosocial action in Pakistan. It is possible that religiosity represents an additional community attachment that is central to Pakistani adolescents' responses to COVID-19. Further, in response to COVID-19, the Pakistani government established a national youth-lead program termed 'Tiger Force' that sought to provide those in need with the basic necessities 
of food and other supplies. Membership to Tiger Force is thought to provide young people with feelings of hope, belongingness, pride, and social status through their commitment to the greater good, which may explain the positive associations found between self-interest and social responsibility values.

Although not the central purpose of this research, findings also demonstrate links between COVID-19 attitudes and subsequent preventative health behaviors. While Oosterhoff and Palmer (2020) found that youth who endorsed greater beliefs about the severity of COVID-19 and all health behaviors, findings from this study found that these beliefs were associated with greater social distancing and news monitoring. It is possible that beliefs about the severity of COVID-19 were more strongly connected with disinfecting and hoarding behaviors earlier in the pandemic and these associations dissipated over time. Future research will need to examine trends in these correlations overtime to better understand the discrepancies in these findings.

\section{Implications for Theory and Practice}

Findings from this study have important implications for both theory and practice. Research on adolescent health and development is modeled based on empirical evidence from mostly White, middle-class youth (Nielsen et al., 2017). While these theories and models may translate to youth from different cultural backgrounds, adopting this assumption without empirical evidence ignores important systematic variability in personal experiences. Results from this study provide necessary context to past research examining the role of community attachments within adolescent public health behavior by highlighting that some of these connections may be specific to the nature of the pandemic and culture. By extending past research to youth from Pakistan, this study provides an initial step in building a culturally sensitive theoretical model of how community attachments may be linked with adolescent health.

Findings also have applied implications for programs in Pakistan seeking to reduce the spread of COVID-19 or future diseases. Specifically, our findings indicate that targeting community attachments may have implications for increasing compliance with government regulations and best practices to reduce the spread of COVID-19 among young people. Messaging campaigns emphasizing social responsibility and social trust may be a reasonable strategy to help increase preventative health behaviors. These findings are especially promising given the rarity of studies on youth in Pakistan despite those under the age of 18 representing approximately $50 \%$ of the population. Such interventions hold promise given that adolescents engage in wide variety of prosocial behaviors during the pandemic (Alvis et al., 2020) and appear to be motivated by helping others (Oosterhoff et al., 2020).

\section{Limitations and Future Directions}

Findings from this study should be interpreted in the context of certain limitations. The research design was cross-sectional and causal or temporal inferences cannot be made. Future research using longitudinal designs is needed to understand temporal ordering of these associations. The sample was recruited using social media, which was advantages given the health and logistical challenges of collecting data with adolescents in Pakistan during a pandemic. However, this method of data collection may be prone to selection 
bias, as evidenced by the greater proportion of female youth in this study. Future research using a wide range of sampling procedures is needed to better understand how social media recruitment may have influenced these findings. Additionally, data collection occurred over a period of one month to gain an adequate sample size. The rapid social changes of the virus may have altered adolescents' community attachments or pandemic-related behaviors. Although consistent with past research (Oosterhoff \& Palmer, 2020) and validated with adolescent samples (Syvertsen et al., 2015), the community attachment measures used in this study contained a small number of items and both hoarding behavior and news monitoring were assessed with single items. Future research would benefit from using expanded measures of community attachments and COVID-19 health behaviors.

\section{Conclusions}

Despite initiatives to broaden public health science beyond Western samples, the majority of research on adolescent development and public health has been conducted in the United States or Europe. Such efforts-while important—provide a limited perspective on adolescent health if not empirically examined within non-Western contexts. Results from this study emphasize that community attachments may play an important role in guiding adolescents' pandemic-related responses in Pakistan. These findings contribute to the limited although growing body of research seeking to understand adolescent health in Pakistan and enrich psychological science by examining these processes in Pakistani youth.

Supplementary Information The online version contains supplementary material available at https://doi. org/10.1007/s10566-021-09657-7.

\section{References}

Alvis, L., Shook, N., \& Oosterhoff, B. (2020). Adolescents' prosocial experiences during the covid-19 pandemic: Associations with mental health and community attachments. Retrieved from: https://psyarxiv. $\mathrm{com} / 2 \mathrm{~s} 73 \mathrm{n}$.

Imran, N., Zeshan, M., \& Pervaiz, Z. (2020). Mental health considerations for children \& adolescents in COVID-19 pandemic. Pakistan Journal of Medical Sciences. https://doi.org/10.12669/pjms.36.COVID 19-S4.2759

Kang, S. H., \& Skidmore, M. (2018). The effects of natural disasters on social trust: Evidence from South Korea. Sustainability, 10, 2973.

Metzger, A., Alvis, L. M., Oosterhoff, B., Babskie, E., Syvertsen, A., \& Wray-Lake, L. (2018). The intersection of emotional and sociocognitive competencies with civic engagement in middle childhood and adolescence. Journal of Youth and Adolescence, 47, 1663-1683.

Nielsen, M., Haun, D., Kärtner, J., \& Legare, C. H. (2017). The persistent sampling bias in developmental psychology: A call to action. Journal of Experimental Child Psychology, 162, 31-38.

Oosterhoff, B., \& Palmer, C. A. (2020). Attitudes and psychological factors associated with news monitoring, social distancing, disinfecting, and hoarding behaviors among US adolescents during the coronavirus disease 2019 pandemic. JAMA Pediatrics, 174, 1184-1190.

Oosterhoff, B., Palmer, C. A., Wilson, J., \& Shook, N. (2020). Adolescents' motivations to engage in social distancing during the COVID-19 pandemic: Associations with mental and social health. Journal of Adolescent Health, 67, 179-185.

Sawyer, S. M., Afifi, R. A., Bearinger, L. H., Blakemore, S. J., Dick, B., Ezeh, A. C., \& Patton, G. C. (2012). Adolescence: A foundation for future health. The Lancet, 379(9826), 1630-1640. 
Syvertsen, A. K., Wray-Lake, L., \& Metzger, A. (2015). Youth civic and character measures toolkit. Search Institute.

Van Bavel, J. J., Baicker, K., Boggio, P. S., Capraro, V., Cichocka, A., Cikara, M., \& Drury, J. (2020). Using social and behavioural science to support COVID-19 pandemic response. Nature Human Behaviour, $4,1-12$.

Wray-Lake, L., Maggs, J. L., Johnston, L. D., Bachman, J. G., O’Malley, P. M., \& Schulenberg, J. E. (2012). Associations between community attachments and adolescent substance use in nationally representative samples. Journal of Adolescent Health, 51, 325-331.

Publisher's Note Springer Nature remains neutral with regard to jurisdictional claims in published maps and institutional affiliations. 